\title{
Transtorno de ansiedade generalizada: uma revisão narrativa
}

\author{
Generalized anxiety disorder: a narrative review
}

Trastorno de ansiedad generalizada: una revisión narrativa

Amanda Brandão Lopes ${ }^{1 *}$, Luisa Lago de Souza1, Lara Ferreira Camacho ${ }^{2}$, Simone Fernandes Nogueira $^{2}$, Ana Carolina Marinho Coelho Vasconcelos ${ }^{2}$, Lays Teixeira de Paula ${ }^{3}$, Matheus de Oliveira Santos $^{4}$, Fernanda Paula Atavila ${ }^{5}$, Gabrielle Fernanda Cerbarro ${ }^{6}$, Rogério Wilson Brandão Fernandes ${ }^{2}$.

\section{RESUMO}

Objetivo: Ampliar os conhecimentos sobre Transtorno de Ansiedade Generalizada (TAG). Revisão bibliográfica: A ansiedade é uma emoção humana considerada normal, no entanto, algumas vezes pode se tornar excessiva e assumir um significado patológico, se tornando um distúrbio psiquiátrico e trazendo prejuízos à vida do indivíduo. O TAG é muitas vezes subdiagnosticadoe tratado de maneira incorreta. As pessoas que apresentam sintomas sugestivos da doença devem procurar um médico psiquiatra para uma avaliação. O diagnóstico é realizado através dos critérios recomendados pelo DiagnosticandStatistical Manual of Mental Disorders V(DSM-V).O tratamento é feito com terapia, principalmente com a Terapia Cognitivo Comportamental (TCC) e/ou medicamentos, preferencialmente com as drogas de primeira linha: Inibidores Seletivos da Recaptação de Serotonina (ISRS) e Inibidores da Receptação de Serotonina e Noradrenalina (IRSN). A combinação de ambos é a melhor opção terapêutica. Considerações finais: $O$ TAG é um distúrbio psiquiátrico extremamente prevalente na sociedade atual e que causa inúmeros problemas sociais. Portanto, deve ser devidamente diagnosticado e tratado afim de evitar complicações do quadro.

Palavras-chave: Ansiedade, Transtorno de ansiedade generalizada, Distúrbios psiquiátricos.

\begin{abstract}
Objective: To increase knowledge about Generalized Anxiety Disorder (GAD). Bibliography review: Anxiety is a human emotion considered normal, however, it can sometimes become excessive and assume a pathological meaning, becoming a psychiatric disorder and harming the individual's life. GAD is often underdiagnosed and treated incorrectly. People who have symptoms suggestive of the disease should see a psychiatrist for an evaluation. Diagnosis is performed using the criteria recommended by the Diagnostic and Statistical Manual of Mental Disorders V (DSM-V). Treatment consists of therapy, mainly Cognitive Behavioral Therapy (CBT) and/or medications, preferably with first-line drugs: Selective Serotonin Reuptake Inhibitors (SSRIs) and Serotonin and Noradrenaline Reuptake Inhibitors (SNRI). The combination of both is the best therapeutic option. Final considerations: GAD is an extremely prevalent psychiatric disorder in today's society that causes numerous social problems. Therefore, it must be properly diagnosed and treated in order to avoid complications of the condition.
\end{abstract}

Keywords: Anxiety, Generalized anxiety disorder, Psychiatric disorders.

\footnotetext{
${ }^{1}$ Faculdade de Minas de Belo Horizonte (FAMINAS-BH), Belo Horizonte - MG.

*E-mail: amandabrandaolopes@gmail.com

2 Faculdade de Ciências Médicas e da Saúde de Juiz de Fora (SUPREMA), Juiz de Fora - MG.

${ }^{3}$ Centro Universitário UNIFAMINAS, Muriaé - MG.

${ }^{4}$ Centro Universitário Presidente Tancredo de Almeida Neves (UNIPTAN), São João Del Rei - MG.

${ }^{5}$ Centro Universitário ITPAC, Palmas - TO.

${ }^{6}$ Faculdade São Leopoldo Mandic, Araras - SP.
} 


\section{RESUMEN}

Objetivo: Incrementar el conocimiento sobre el Trastorno de Ansiedad Generalizada (TAG). Revisión bibliográfica: La ansiedad es una emoción humana considerada normal, sin embargo, en ocasiones puede volverse excesiva y asumir un significado patológico, convirtiéndose en un trastorno psiquiátrico y perjudicando la vida del individuo. EI TAG a menudo se infradiagnostica y se trata de manera incorrecta. Las personas que presenten síntomas que sugieran la enfermedad deben consultar a un psiquiatra para una evaluación. El diagnóstico se realiza siguiendo los criterios recomendados por el Manual Diagnóstico y Estadístico de los Trastornos Mentales V (DSM-V). El tratamiento consiste en terapia, principalmente Terapia Cognitivo-Conductual (TCC) y / o medicamentos, preferiblemente con fármacos de primera línea: Inhibidores Selectivos de la Recaptación de Serotonina (ISRS) e Inhibidores de la Recaptación de Serotonina y Noradrenalina (ISRS). La combinación de ambos es la mejor opción terapéutica. Consideraciones finales: EI TAG es un trastorno psiquiátrico extremadamente prevalente en la sociedad actual que causa numerosos problemas sociales. Por lo tanto, debe diagnosticarse y tratarse adecuadamente para evitar complicaciones de la afección.

Palabras clave: Ansiedad, Trastorno de ansiedad generalizada, Trastornos psiquiátricos.

\section{INTRODUÇÃO}

Apesar de na grande maioria das vezes, ser considerada uma emoção humana normal, a ansiedade pode se tornar um distúrbio psiquiátrico quando aparece de forma exacerbadae acaba por assumir um significado patológico. Quando isso ocorre, a ansiedade pode afetar consideravelmente as Atividades Instrumentais de Vida Diária (AIVD), sendo classificado como um transtorno emocional (ZUARDI AW, 2017).

É muito comum a associação entre a ansiedade e os transtornos depressivos, principalmente no que diz respeito aos transtornos de ansiedade generalizada e aos transtornos de pânico. Isso muitas vezes pode dificultar o diagnóstico eo tratamento, tornando-os mais complexos para os médicos não especialistas. Sendo assim, os transtornos de ansiedade são, por muitas vezes, subdiagnosticados e subtratados na atenção primáriade saúde (ROSE GM e TADI P, 2021).

O medo desproporcional, a preocupação excessiva e uma sensação corriqueira de estar sobrecarregado são alguns sinais que podem estar presentes noTranstorno de Ansiedade Generalizada (TAG). Nesses pacientes a preocupação é caracterizada como persistente, excessiva e irreal com relação as atividades diárias, podendo ser multifocal, envolvendo futuro, família, finanças e saúde. É de difícil controle e pode estar associado a muitos sintomas físicos e psicológicos não específicos. Essa preocupação é considerada a característica central do TAG (GOTTSCHALK MG, 2017).

O tratamento para os transtornos de ansiedade engloba terapia psicológica e farmacoterapia, sendo a combinação de ambas uma proposta terapêutica com melhores resultados. A psicoterapia considerada com maior nível de evidência é a Terapia Cognitivo-Comportamental (TCC). Os Inibidores Seletivos da Recaptação da Serotonina (ISRS) e os Inibidores da Recaptação da Serotonina e Norepinefrina (IRSN) são considerados os medicamentos de primeira linha (NATIONAL INSTITUTE FOR HEALTH AND CARE EXCELLENCE (NICE), 2019).

Outras opções de medicamentosas incluem antidepressivos tricíclicos, pregabalina, moclobemida ebuspirona. Os benzodiazepínicos, por sua vez, não são recomendados para uso rotineiro. No momento de traçar um plano terapêutico, o médico deve levar em consideração a eficácia, o custo, as interações, os efeitos adversos das medicações e após a remissão do quadro, o tratamento deve ser mantido de forma contínua por cerca de 6 a 12 meses afim de garantir um controle mais eficaz do distúrbio (MARON E, 2017).

Essa revisão teve por objetivo fornecer uma ampla abordagem sobre os transtornos de ansiedade, uma vez que são extremamente prevalentes na sociedade atual. 


\section{REVISÃO BIBLIOGRÁFICA}

\section{Epidemiologia do transtorno de ansiedade generalizada}

O número de pessoas que sofrem de transtornos de ansiedade teve um aumento de cerca de $15 \%$ desde 2005, segundo a Organização Mundial da Saúde (OMS), sendo cerca de 264 milhões de pessoas globalmente afetadas. Devido à sua alta prevalência, a ansiedade pode ter uma carga de custo maior quando comparado a outros transtornos psiquiátricos, podendolevar a ausências no trabalho e na escola (ROSE GM e TADI P, 2021).

Dentre os transtornos de ansiedade, os mais comuns, em ordem decrescente de prevalência são: as fobias específicas, o transtorno do pânico, fobia social e ansiedade generalizada. Suas respectivas taxas de incidência são 10,3\%, 6,0\%, 2,7\% e 2,2\%. De modo geral, as mulheres são mais propensas a desenvolver distúrbios emocionais do que os homens, com início principalmente no período da adolescência (NICE, 2019).

\section{Fatores de risco para o transtorno de ansiedade generalizada}

Com exceção do transtorno obsessivo-compulsivo (TOC), as mulheres costumam ter cerca de duas vezes mais probabilidade de apresentarem transtornos de ansiedade durante a vida do que os homens. Eles podem aparecer em qualquer idade sendo, no geral, mais comum na adolescência. Existem algumas condições sociais que aumentam os riscos desses transtornos, sendo elas: estado civil solteiro, viúvo ou divorciado, isolamento social e falta de apoio de amigos ou familiares. O trauma psicológico também é um fator que pode desencadear transtornos de ansiedade, sendo o transtorno de estresse pós-traumático o mais comum em pessoas vulneráveis (ZUARDI AW, 2017).

Existem alguns transtornos de ansiedade que são considerados mais comuns na infância, sendo eles as fobias simples, como a ansiedade de separação, o medo do escuroe 0 transtorno obsessivocomportamental. As crianças afetadas podem acabar desenvolvendo depressão, uso abusivo de inúmeras substâncias na idade adulta, como por exemplo bebidas alcóolicas ou outros transtornos de ansiedade. Normalmente a ansiedade infantil ocorre entre os 13 e os 18 anos em cerca de 1 em cada 4 crianças, sendo a idade média de início dos sinais e sintomas a partir dos11 anos. A prevalência de um transtorno de ansiedade grave ao longo da vida em crianças entre os 13 e os 18 anos de idade é de aproximadamente $6 \%$ e a prevalência geral em menores de 18 anos é entre 5,7\% e 12,8\% (NICE, 2019).

\section{Etiologia e fisiopatologia do transtorno de ansiedade generalizada}

A etiologia dos transtornos de ansiedade é baseada em uma interação de fatores psicossociais e ambientaise também pode está associada a uma vulnerabilidade genética, que se manifesta em disfunções neuropsicológicas e neurobiológicas. Dentre esses fatores podemos citar: estresse, adversidade infantil, traumas, história familiar positiva para doenças psiquiátricas em parentes de $1^{\circ} \mathrm{grau}$, existência de comorbidades, abuso infantil, entre outros (ROSE GM e TADI P, 2021).

A fisiopatologia do transtorno de ansiedade generalizada ainda não tem seu mecanismo totalmente conhecido. Entre cerca de 7 a 9 meses de vida, as crianças começam a estranhar as pessoas que não estão no seu ciclo de vida diário, o que pode gerar uma ansiedade podendo ser considerada um fenômeno normal na infância. Os sistemas serotonérgicos, noradrenérgicos e outros sistemas de neurotransmissores parecem desempenhar um papel de extrema importância na resposta do corpo ao estresse e são via comumente afetadas na ansiedade. O TAG está muito associadoa uma baixa atividade do sistema serotoninérgico e a uma elevada atividade do sistema noradrenérgico. Sendo assim, os ISRS e os IRSNos medicamentos considerados como primeira linha para o tratamento desse distúrbio psiquiátrico (MARON E, 2017).

\section{Diagnóstico do transtorno de ansiedade generalizada segundo o DSM-V}

A grande maioria dos pacientes comdiagnóstico de transtorno de ansiedade apresenta o início dos sintomas antes dos 20 anos de idade começando normalmente desde a primeira infância e a adolescência. Em muitos casos os pacientes acreditam que a ansiedade social éparte da sua personalidade e, portanto, 
não requer tratamento específico, o que faz com que o TAG seja muitas vezessubdiagnosticado e subtratado. Normalmente esses pacientes só procuram por ajuda médicaquando há presença de outros distúrbios como a depressão maior, o uso abusivo de substâncias e outros transtornos relacionados (MUNIR S e TAKOV V, 2021).

Existem emoções habituais como o medo e a ansiedade que geram as principais características do TAG. O sofrimento e o prejuízo relacionados as atividades básicas diárias devem sempre ser avaliados nesses pacientes. Sendo assim, é feito o diagnóstico de transtorno de ansiedade quando há sofrimento ou prejuízo no funcionamento social, profissional ou em qualquer outro âmbito da vida do indivíduo. $\mathrm{O}$ uso abusivo de substâncias, como álcool ou drogas, assim como algumas condições médicas podem gerar sentimento demedo e ansiedade e, por isso, é importante afastar essa possibilidade no momento do diagnóstico, sendo essas manifestações explicadas por esses fatores e não por um distúrbio de neurotransmissores (CROCQ MA, 2017).

É importante também investigar a história pregressa do paciente para identificar episódios de ansiedade semelhantes no passado ou outros eventos de doenças psiquiátricas. Apesar de não ser um ponto que consta nos critérios diagnósticos, é bastante útil do ponto de vista clínico. O diagnóstico de ansiedade ganha mais força quando o paciente relata a existência de sintomas desde a infância e também a presença de transtornos psiquiátricos em familiares (ONTARIO HQ, 2017).

Durante a formulação do Manual Diagnóstico e Estatístico de Transtornos Mentais, quinta edição (DSMV), foi bastante conturbada a relação entre TAG e transtornos depressivos. Existem estudos que explicam essa relação por interações genéticas, que demonstram que o TAG e a depressão não bipolar podem representar diferentes expressões fenotípicas de uma etiologia comum. Dessa forma, houve a proposta de fundir o TAG e a depressão não bipolar em um único espectro de transtornos de humor e/ou ansiedade, sendo criadas três subclasses de distúrbios emocionais: os transtornos bipolares; os transtornos de angústia (transtorno depressivo maior, transtorno de estresse pós-traumático, TAG e transtorno distímico); transtornos do medo (síndrome do pânico, fobia social, agorafobia e fobia específica) (CROCQ M, 2017).

Os critérios de diagnóstico presentes no DSM-V são fundamentais para o diagnóstico final de transtorno de ansiedade generalizada e eles incluem: a presença de ansiedade e preocupação excessivas por pelo menos 6 meses; a ansiedade está associada a três ou mais dos sintomas a seguir por pelo menos 6 meses: inquietação, ficar facilmente cansado, sensação de tensão ou nervosismo, irritabilidade, tensão muscular, dificuldade de concentração ou sensação de mente em "branco" e/ou distúrbios de sono;a dificuldade em controlar as preocupações; a ansiedade resulta em sofrimento significativo ou prejuízo nas áreas sociais e ocupacionais; a ansiedade não é atribuível a nenhuma causa física ou medicamentosa. Quando esses critérios são preenchidoso indivíduo é então diagnosticado com TAG e rapidamente deverá ser instituída uma proposta terapêutica (SAAED SA, et al., 2019).

Como diagnósticos diferenciais de TAG podem ser citados o feocromocitoma, a DPOC, o hipertireoidismo, a epilepsia, o transtorno bipolar e o uso de descongestionantes, cafeína e albuterol. Esses fatores podem gerar sintomas semelhantes aos de transtorno de ansiedade. $O$ transtorno de ansiedade social deve ser diferenciado de outros transtornos, como transtorno do pânico, agorafobia, transtorno do espectro do autista, transtornos depressivos, transtorno dismórfico corporal, transtornos relacionados a substâncias e vícios e transtornos de personalidade. Hikikomori é uma outra possibilidade que deve ser descartado, uma vez que se tratade uma forma extrema de isolamento social que se estende por mais de 6 meses (SAPRA A, et al.,2020).

\section{Tratamento do transtorno de ansiedade generalizada}

Diante de um diagnóstico de transtorno de ansiedade generalizada, os dois principais tratamentos indicados são as terapias e os medicamentos. Normalmente a maioria dos pacientes acometidosse beneficiam da combinação de ambos. A terapia cognitivo-comportamental, a terapia de suporte e a terapia interpessoal são os três tipos mais comuns de psicoterapia para o tratamento do TAG (GARAKANI A, et al., 2020). 
Na terapia cognitivo-comportamental o principal objetivo envolvea conscientização dos pacientes de que existem alguns pensamentos, expectativas, atitudes e crenças negativas automáticas que contribuem significativamente para o surgimento dos sentimentos de tristeza e ansiedade. Os pacientes aprendem como surgiram e como funcionam esses padrões de pensamento, que podem inclusive terem se desenvolvido no passado para lidar com experiências difíceis ou dolorosas, como podem ser identificados e o que fazer para alterá-los afim de reduzir a infelicidade gerada pela ansiedade (SAAED SA, et al., 2019).

A terapia interpessoal tem como objetivo identificar e solucionar problemas existentes no estabelecimento e na manutenção de relacionamentos satisfatórios. Alguns desses problemas incluem lidar com perdas, conflitos, mudanças de vida e mais facilidade em determinadas situações sociais. Geralmente a terapia de suporte é uma terapia não estruturada que foca nas habilidades interpessoais básicas do terapeuta, como incentivo, escuta empática e reflexão (STRAWN JR, et al., 2018).

É considerado como um tratamento psicológico no qual o terapeuta se estrutura apenas na escuta ativa e na oferta de apoio, com foco nos problemas e nas preocupações dos pacientes, geralmente ele não se engaja em nenhuma outra estratégia terapêutica. Os problemas atuais são muito mais o foco da consulta do que as dificuldades de longo prazo, sendo assim, o objetivo geral é diminuir o nível de desconforto dos pacientes e para que possam enfrentar as circunstâncias atuais com mais facilidade (HALL J, et al., 2019).

Para tratar o transtorno de ansiedade generalizada existem, no tratamento farmacológico, diversos tipos de medicamentos que podem ser usados. Dentre os medicamentos de primeira linha estão as classes de inibidores seletivos da recaptação da serotonina e inibidores da recaptação da serotonina-norepinefrina. Os antidepressivos atípicos também podem ser usados para o tratamento (SAAED SA, et al., 2019).

Dentre os ISRS estão o escitalopram, a sertralina, a paroxetina, a fluoxetina eo citalopram. Dentre os IRSN estão a duloxetina e a venlafaxina. Já dentre os antidepressivos atípicos estão a mirtazapina e o vilazodone. Outros medicamentos que também podem ser usados são os antidepressivos tricíclicos como a clomipramina e a imipramina, além de medicamentos como a pregabalina, trazodona, buspirona e benzodiazepínicos. Os antipsicóticos também podem auxiliar o tratamento de alguns pacientes, em especial aqueles com problemas de comportamento associados. Em casos resistentes, o tratamento combinado pode ser uma opção terapêuticaeficaz (MUSCATELLO MRA, et al., 2019).

Quando é desejada uma redução imediata dos sintomas ou é necessário um tratamento de curto prazo, os benzodiazepínicos como o clonazepam, o alprazolam e o diazepam são agentes utilizados. Os pacientes que são cooperativos e complacentes e estão cientes de que seus sintomas têm uma base psicológica normalmente possuem maior probabilidade de responder bem aos benzodiazepínicos. Os pacientes com histórico de alcoolismo ou abuso de drogas não são candidatos adequados para esse tratamento, uma vez que existe a preocupação com o seu uso indevido e a dependência (BANDELOW B, 2017).

Os ISRS inibem o transportador de recaptação de serotonina (5-hidroxitriptamina, 5-HT) e, em alguns casos, inibem fracamente os mecanismos de recaptação de dopamina e norepinefrina. A inibição da recaptação de 5-HT aumenta as concentrações de 5-HT sináptica, que por sua vez aumenta a difusão extra-sináptica. Os ISRS diferem significativamente em termos de potência e seletividade para 0 transportador 5-HT em relação aos transportadores de norepinefrina e dopamina, bem como em sua capacidade de interagir com outros receptores sinápticos e extra-sinápticos, o que subtende sua eficácia variável e perfis de efeitos colaterais. Além disso, todos esses compostos diferem em seu metabolismo, perfis de efeitos colaterais e duração da ação (STRAWN JR, et al., 2018).

O efeito ansiolítico desses antidepressivos normalmente possuem um período de latência que pode variar entre 2 e 4 semanas, em alguns casos chegando até 6 semanas e os pacientes devem ser informados sobre isso antes de iniciar o tratamento. Além disso, é importante orientar que os efeitos adversos podem ser mais evidentes durante as primeiras 2 semanas, podendo ocorrer tremor inicial ou um aumento nos sintomas de ansiedade, o que pode reduzir a adesão do paciente ao tratamento se não for muito bem explicado. Estes efeitos adversos podem ser reduzidos com a diminuição da dose inicial dos antidepressivos. De acordo com a experiência clínica, a tolerabilidade pode diferir entre os pacientes, e também é possível que um paciente individual possa experimentar menos efeitos adversos ao mudar de uma classe medicamentosa para outra (GARAKANI A, et al., 2020). 
Alguns medicamentos dessas classes farmacológicas são inibidores das enzimas do citocromo P450 e, portanto, podem interagir com outras drogas psicofarmacológicas e medicamentos para outras condições clínicas. Após a interrupção do tratamento com um ISRS, podem ocorrer reações de abstinência. Essas reações são muito menos frequentes e graves do que as observadas após o término do tratamento com benzodiazepínicos e podem ser mais frequentes com a paroxetina do que com a sertralina ou com a fluoxetina (HALL J, et al., 2019).

O medicamento é geralmente bem tolerado e considerado seguro em todas as indicações aprovadas em adultos, embora os benefícios terapêuticos potenciais de altas doses estejam associados a efeitos colaterais emergentes, sem redução significativa dos sintomas e taxas de remissão final. As reações adversas mais comumente relatadas foram:boca seca, náusea, constipação, diminuição do apetite, sonolência e hiperidrose, que ocorreram principalmente nos estágios iniciais e desapareceram após as primeiras semanas de tratamento.Na terapia de longo prazo, com uso por cerca de 6 meses a 1 ano, os efeitos adversos emergentes do tratamento que foram frequentemente observados em pacientes adultos incluem: visão turva,palpitações, ganho ou perda de peso,vertigem, calafrios e prurido (BANDELOW B, 2017).

\section{Seguimento do paciente com transtorno de ansiedade generalizada}

O acompanhamento mensal é previsto pelas psicoterapias. $E$, no caso de um tratamento medicamentoso, recomenda-se que seja agendada uma consulta de retorno para a semana seguinte ao início do tratamento a fim de avaliar a adesão, monitorar a resposta e os efeitos adversos relacionados ao uso do fármaco (UNIVERSIDADE FEDERAL DO RIO GRANDE DO SUL, 2017).

Uma nova reavaliação é indicada por volta da $4^{\text {a }}$ semana de tratamento e, em caso de remissão dos sintomas, são sugeridas consultas mensais. Depois da estabilização do quadro, com melhora alcançada a partir do $4^{\circ}$ mês, as consultas podem ser espaçadas para um período de dois em dois meses. O período de manutenção pode variar de 6 a 24 meses de uso contínuo da medicação e, posteriormente, ela deverá ser gradualmente retirada e a resposta do paciente à suspensão do fármaco deve ser acompanhada de perto (UNIVERSIDADE FEDERAL DO RIO GRANDE DO SUL, 2017).

\section{Prognóstico e complicações do transtorno de ansiedade generalizada}

Caso não seja tratado de forma adequada, o TAG, caracterizado como um transtorno debilitante e altamente prevalente, pode acabar resultando em pior desempenho ocupacional, menor escolaridade, interação social prejudicada, diminuição geral da qualidade de vida do indivíduo e relacionamentos de baixa qualidade. O prognóstico para pacientes com TAG é variante, uma vez que alguns pacientes não aderem ao tratamento devido ao custo elevado e aos efeitos adversos dos medicamentos. As recaídas são comuns e os pacientes costumam procurar novamente o médico para controle da doença (MUSCATELLO MRA, et al., 2019).

Dentre as complicações do TAG está a possibilidade desse distúrbio levar, ou piorar, outras condições físicas e mentais como insônia, uso indevido de drogas ou álcool, depressão, isolamento social, problemas de funcionamento no trabalho ou escola resultando em uma qualidade de vida prejudicada. Além disso, esses pacientes possuem um alto potencial de suicídio (HALL J, et al., 2019).

\section{CONSIDERAÇÕES FINAIS}

O TAG é um distúrbio psiquiátrico extremamente prevalente na sociedade atual. É caracterizado por sintomas de preocupação persistente, excessiva e irreal com relação as atividades básicas diárias, o que resulta em uma queda da qualidade de vida do indivíduo. O diagnóstico deve ser realizado preferencialmente por um médico psiquiatra seguindo os critérios do DSM-V. O tratamento é feito com terapia, principalmente a TCC e/ou com medicamentos, sendo as drogas de primeira linha os ISRS e os IRSN. A combinação de ambos é a terapêutica que apresenta melhor resultado nessas pessoas. O paciente deve ser acompanhado até a remissão completa da doença, com o objetivo de evitar complicações como desenvolvimento de uma depressão grave e suicídio. 


\section{REFERÊNCIAS}

1. BANDELOW B, et al. Treatment of anxiety disorder. Dialogues Clinical Neuroscience, 2017; 19(2):93-107.

4. CROCQ MA. The history of generalized anxiety disorder as a diagnostic category. Dialogues Clinical Neuroscience, 2017; 19(2):107-116.

5. GARAKANI A, et al. Pharmacotherapy of Anxiety Disorders: Current and Emerging Treatment Options. Frontiers in Psychiatry, 2020; 11:595584.

6. GOTTSCHALK MG. Genetics of generalized anxiety disorder and related traits. Dialogues Clinical Neuroscience, $2017 ; 19(2): 159-168$.

7. HALL J, et al. Efficacy of Cognitive Behavioral Therapy for Generalized Anxiety Disorder in Older Adults: Systematic Review, Meta-Analysis, and Meta-Regression. The American Journal of Geriatric Psychiatry, 2016; 24(11):10631073.

8. MARON E. Biological markers of generalized anxiety disorder. Dialogues Clinical Neuroscience, 2017; 19(2):147158.

9. MUNIR S, TAKOV V. Generalized anxiety disorder. StatPearls, 2021; 1(2):1-4.

10. MUSCATELLO MRA, et al. Duloxetine in Psychiatric Disorders: Expansions Beyond Major Depression and Generalized Anxiety Disorder. Frontiers in Psychiatry, 2019; 10(1):772.

11. NATIONAL INSTITUTE FOR HEALTH AND CARE EXCELLENCE (NICE). Nice Guidiline. Generalised anxiety disorder and panic disorder in adults: management. Subject to Notice of rights, United Kingdom, 2019 ; 1-41. Disponível em: https://www.nice.org.uk/guidance/cg113/resources/generalised-anxiety-disorder-and-panic-disorderin-adults-management-35109387756997. Acesso em: 03 set 2021.

12. ONTARIO HQ. Psychotherapy for Major Depressive Disorder and Generalized Anxiety Disorder: A Health Technology Assessment. Ontario Health Technology Assessment Series, 2017; 17(15):1-167.

13. ROSE GM, TADI P. Social anxiety disorder. StatPearls, 2021; 1(2):1-7.

14. SAAED SA, et al. Depression and Anxiety Disorders: Benefits of Exercise, Yoga, and Meditation. American Family Physiciam, 2019; 99(10):620-627.

15. SAPRA A, et al. Using Generalized Anxiety Disorder-2 (GAD-2) and GAD-7 in a Primary Care Setting. Cureus Publishing Beyond Open Access, 2020; 12(5):e8224.

16. STRAWN JR, et al. Pharmacotherapy for Generalized Anxiety Disorder in Adults and Pediatric Patients: An Evidence-Based Treatment Review. Expert Opinion on Pharmacotherapy. 2018; 19(10):1057-1070.

17. UNIVERSIDADE FEDERAL DO RIO GRANDE DO SUL. TeleConduta: Ansiedade. TelessaúdeRS-UFRGS, 2017 ; 1 $14 . \quad$ Disponível em: https://www.ufrgs.br/telessauders/documentos/telecondutas/Telecondutas_Ansiedade_20170331.pdf. Acesso em: 03 set 2021.

18. ZUARDI AW. Características básicas do transtorno deansiedade generalizada. Revista USP, 2017; 50(1):51-55. 